\title{
A Hybrid Density Functional Study on PuN
}

\author{
RONG YANG ${ }^{a}$, BIN TANG $^{b, *}$ AND TAO GAO ${ }^{c}$ \\ ${ }^{a}$ College of Materials Science and Engineering, Chongqing Jiaotong University, Chongqing 400074, PR China \\ ${ }^{b}$ School of Business Administration, Chongqing City Management College, Chongqing 401331, PR China \\ ${ }^{c}$ Institute of Atomic and Molecular Physics, Sichuan University, Chengdu 610065, PR China
}

(Received May 21, 2017; in final form November 2, 2017)

The structural, magnetic, electronic, dynamical and thermodynamic properties of PuN have been studied within the frameworks of the density-functional theory, DFT $+U$ and hybrid DFT. The calculations have been performed using the full-potential-linearized augmented plane-wave method. The ferromagnetic and antiferromagnetic configurations are considered in this work. The lattice constants, bulk moduli, densities of states, and thermodynamic data have been computed and compared to available experimental data and other theoretical calculations published in the literature. Total energy results obtained with DFT $+\mathrm{U}$ and hybrid DFT indicate that the ground state of $\mathrm{PuN}$ is antiferromagnetic, in agreement with experiment. The chemical bonds between $\mathrm{Pu}$ and $\mathrm{N}$ have a mixture of covalent and ionic components, but the ionic character is stronger than covalent character. The phonon dispersion curves and the optical-mode frequencies are also reported. At last, the effect of spin-orbit coupling on the structural, magnetic, and electronic properties of PuN has been discussed.

DOI: 10.12693/APhysPolA.133.32

PACS/topics: plutonium mononitride, density-functional theory, hybrid functional

\section{Introduction}

Actinide nitrides are of interest due to their prospective applications [1-3]. Actinide nitrides are not only very promising advanced fuel materials for certain generation IV reactors, but also target materials for the transmutation of plutonium and minor actinides in fast reactor cores and in accelerator driven systems. Therefore, it is of crucial importance to model the structural and physical properties of actinide nitrides, because it can provide valuable information concerning fuel performance and stability.

Of actinide-nitrogen compounds, plutonium forms only the mononitride $(\mathrm{PuN})[4-5]$. This compound has the simple rock-salt-type structure. To PuN, some fundamental characteristics are known already. Some experimental data on structural parameters [6] and thermodynamic properties [7] can be obtained. Antiferromagnetic ordering at $13 \mathrm{~K}$ was suggested by Martin et al. [8] on the basis of a maximum in magnetic susceptibility and specific heat. A photoelectron spectroscopy experiment on PuN was performed by Havela et al. [9]. The photoelectron spectroscopy study reveals that the $5 f$ states appear in the vicinity of the Fermi level, exhibiting the same type of features as in the majority of $\mathrm{Pu}$ systems studied. In theory, Fynn and Ray [10] investigated the structural, electronic, and magnetic properties of $\mathrm{PuN}$ using the full-potential all-electron linearized augmented plane wave (FP-LAPW) plus local orbital method. Their calculations indicated that the ground state of $\mathrm{PuN}$ is ferromagnetic, which is contrary to Martin et al. [8] conclusion. The ground-state electronic structure of $\mathrm{PuN}$ was

*corresponding author; e-mail: tangbinre@126.com studied by Petit et al. [11] by using the self-interaction corrected local spin-density approximation. They concluded that the localized $5 f^{3}$ configuration is the most probable ground state of $\mathrm{PuN}$, which was questioned by Havela et al. [9] photoelectron spectroscopy experiment. Wen et al. [12] reported the structural, electronic and magnetic properties of PuN by using the Heyd-ScuseriaErnzerhof hybrid functional. They predicted $\mathrm{PuN}$ to be antiferromagnetic. Obviously, there are disagreements in the published literature about plutonium mononitride.

In this paper, the focus is on the structural, magnetic, electronic, dynamical and thermodynamic properties of PuN where we use the density-functional theory (DFT), $\mathrm{DFT}+\mathrm{U}$ and hybrid DFT calculations. Electronic structure is the fundamental factor to determine the physical properties of the material. Especially, it is a complicated challenge to describe the character of the $5 f$ states in PuN. The magnetic properties of PuN need to be carefully examined, since magnetism affects not only the ground state energy of the system, but also the structure of the system. Thus, we try to re-consider the magnetic properties and electronic structure of $\mathrm{PuN}$ in this paper. The lattice vibrations which are critical for understanding phase stability and thermal transport must be obtained. Since measuring the phonons of $\mathrm{PuN}$ is difficult and required experimental data are not available, the theoretical study on phonon spectrum of $\mathrm{PuN}$ is necessary. However, the theoretical study of phonon spectrum is still lacking. Therefore we will explore the lattice dynamical of PuN. From the accurate determination of phonon dispersion curves, we can acquire the useful thermodynamic data, such as the Helmholtz free energy, the specific heat, vibrational entropy and thermal expansion etc. These thermodynamic data must be known in order to properly utilize fuels in a reactor. Here we will also explore the chemical bonding of $\mathrm{PuN}$ for first time. The investigation on the chemical bonding can deepen 
the understanding of the electronic structure of $\mathrm{PuN}$.

To take account of strong correlation effects on $f$ orbital electrons, more and more first principles techniques from the traditional DFT to modern correction schemes such as DFT $+\mathrm{U}$ and hybrid DFT have been performed to investigate the physical properties of $\mathrm{Pu}-$ bearing materials. In this work, hybrid DFT implemented within the FP-LAPW method has been applied to $\mathrm{PuN}$. In order to compare with the results by hybrid DFT, DFT and DFT $+\mathrm{U}$ methods have also been employed. In addition, the effect of spin-orbit coupling (SOC) on $\mathrm{Pu} 5 f$ states will be considered in this work, since the SOC effect plays a pivotal role for $\mathrm{Pu} 5 f$ electrons $[13,14]$.

In this work, we perform DFT, DFT $+\mathrm{U}$ and hybrid DFT calculations to investigate the structural, magnetic, electronic, dynamical and thermodynamic properties of PuN using the FP-LAPW method. The paper is organized as follows. In Sect. 2, we introduce the computational details. In Sect. 3, we first investigate the crystal structure and magnetic properties of $\mathrm{PuN}$. Then, we study the electronic structure and chemical bonding of $\mathrm{PuN}$. In addition, the phonon and thermodynamic properties of PuN are given. At last, the effect of SOC on the structural, magnetic, and electronic properties of $\mathrm{PuN}$ will be discussed. In Sect. 4, we summarize our main findings and draw some conclusions.

\section{Computational details}

All our calculations are solved using the all-electron FP-LAPW method as implemented in the WIEN2k code [15]. Within the FP-LAPW method, the unit cell is divided into non-overlapping muffin tin spheres and an interstitial region. The muffin-tin radii $R_{M T}$ of $\mathrm{Pu}$ and $\mathrm{N}$ atoms have been chosen to be 2.3 a.u. and 1.6 a.u., respectively. The parameter $R_{M T} K_{\max }=8$ is used for the plane waves expansion of the wave function in the interstitial region. Here $K_{\max }$ is the truncation for the modulus of reciprocal lattice vector. The spin-polarized calculations are performed in the WIEN2k code by simple replication of some steps for spin-up and spin-down electrons separately $[16,17]$. The ferromagnetic $(F M)$ and antiferromagnetic (AFM) configurations are considered in this work. We construct a unit cell with four atoms per cell to study the $1 k$ AFM configuration, consisting of alternating spin-up and spin-down ferromagnetic sheets along the [001] direction. The Brillouin zone are sampled with $200 k$-points. For all the iterations, convergence criteria for the charge, total energy and force are $0.001 e$, $0.001 \mathrm{Ry}$ and $0.001 \mathrm{Ry} /$ a.u., respectively.

The exchange-correlation potential is computed with the generalized gradient approximation (GGA) and the local density approximation (LDA). In the $\mathrm{DFT}+\mathrm{U}$ scheme, the value of $\mid U$ is taken as $4.0 \mathrm{eV}$, as determined by Wen et al. [12]. In this work, two hybrid functionals (PBE0 and Fock-0.25) are applied but only to the $5 f$ electrons of $\mathrm{Pu}$ atom. Because we do not believe that applying the hybrid functional to all electrons including $s$ - and $p$-electrons will affect the conclusions of this work. Such functionals are known as exact exchange for correlated electrons (EECE) functionals [18, 19]. The PBE0 $[20,21]$ hybrid functional used in this work is

$$
\begin{aligned}
& E_{x c}^{P B E 0}[\rho]=E_{x c}^{P B E}[\rho]+\frac{1}{4}\left(E_{x}^{H F}\left[\Psi_{\text {sel }}\right]\right. \\
& \left.-E_{x}^{P B E}\left[\rho_{\text {sel }}\right]\right) .
\end{aligned}
$$

The Fock-0.25 hybrid functional used in this work is the one proposed by Moreira et al. [22]:

$$
\begin{aligned}
& E_{x c}^{F o c k-0.25}[\rho]=E_{x c}^{L D A}[\rho]+0.25\left(E_{x}^{H F}\left[\Psi_{\text {sel }}\right]\right. \\
& \left.\quad-E_{x}^{L D A}\left[\rho_{\text {sel }}\right]\right),
\end{aligned}
$$

where $\Psi_{\text {sel }}$ is the wave function of the selected electrons, and $\rho_{\text {sel }}$ is the corresponding electron density of the selected electrons. It is obvious that the form of Eq. (2) is the same as Eq. (1), but LDA replacing PBE. Compared to $\mathrm{DFT}+\mathrm{U}$, hybrid DFT does not contain any system-dependent parameters, in contrast to choosing $U$ in $\mathrm{DFT}+\mathrm{U}$. The difference make hybrid DFT more appealing.

In the spin-orbit coupling calculations, semi-core and valence states are treated at the fully relativistic level. Spin-orbit interaction for semi-core and valence states is incorporated via the second variation approach [23] using the scalar relativistic eigenstates as basis, where all eigenstates with energies below 4.5 Ry are included. To account for the finite character of the $p_{1 / 2}$ wave function at the nucleus, relativistic $p_{1 / 2}$ orbitals have been included in our spin-orbit coupling calculations.

\section{Results and discussion}

\subsection{Crystal structure and magnetic properties}

PuN has a NaCl-type structure. The occupied Wyckoff positions are $4 \mathrm{a}$ of $\mathrm{Pu}$ and $4 \mathrm{~b}$ of $\mathrm{N}$. The known experimental lattice constant of $\mathrm{PuN}$ is $4.905 \AA[6]$. AFM ordering at $1 \mathrm{~K}$ is found in experiment [8]. From the values of energy difference $\Delta E$ between the FM and AFM phases given in Table I, we can see that the total energies of the FM phase are lower in the DFT (LSDA/PBE) method. This indicates that the FM state is more stable than the AFM state in the DFT (LSDA/PBE) method, which is not consistent with the available experimental result [8]. On the other hand, $\mathrm{DFT}+\mathrm{U}(\mathrm{LSDA}+\mathrm{U} / \mathrm{PBE}+\mathrm{U})$ and hybrid DFT (Fock-0.25/PBE0) predict PuN to be AFM, in agreement with experiment. The results imply that the strong correlation effects on magnetism for PuN are significant.

Comparing the calculated and experimental lattice constants in Table I, we can find that all GGA $(\mathrm{PBE} / \mathrm{PBE}+\mathrm{U} / \mathrm{PBE} 0)$ calculations overestimate the lattice constants in the FM/AFM state. It may indicate that GGA calculations overestimate the electron localization and underestimate the corresponding contribution to bonding for $\mathrm{PuN}$. On the whole, the lattice constants predicted by the LDA (LSDA/LSDA $+\mathrm{U} /$ Fock-0.25) scheme 
TABLE I

Compilation of data on FM and AFM states of PuN: lattice constant $a[\AA]$, total magnetic moment $\mu_{t o t}$, band gap $E_{\text {gap }}[\mathrm{eV}], \Delta E=E_{F M}-E_{A F M}[\mathrm{eV} /$ per PuN] and bulk modulus $B[\mathrm{GPa}]$. The Hubbard parameter $U$ is $0.29 \mathrm{Ry}$.

\begin{tabular}{c|c|c|c|c|c|c}
\hline \hline Method & magn. & $a[\AA]$ & $\mu_{\text {tot }}\left[\mu_{\mathrm{B}}\right]$ & $E_{\text {gap }}[\mathrm{eV}]$ & $\Delta E[\mathrm{eV}]$ & $B[\mathrm{GPa}]$ \\
\hline \multirow{2}{*}{ LSDA } & FM & 4.815 & 4.504 & 0.0 & -0.09 & 178 \\
& AFM & 4.801 & 0.000 & 0.0 & 0.00 & 187 \\
LSDA+U & FM & 5.035 & 4.943 & 0.0 & 0.22 & 185 \\
& AFM & 4.916 & 0.000 & 0.0 & 0.00 & 172 \\
Fock-0.25 & FM & 4.851 & 3.750 & 0.0 & 0.10 & 208 \\
PBE & AFM & 4.876 & 0.000 & 0.0 & 0.00 & 221 \\
& FM & 4.944 & 4.881 & 0.0 & 0.00 & 146 \\
PBE+U & AFM & 4.926 & 0.000 & 0.0 & 0.13 & 158 \\
& FM & 5.103 & 5.000 & 0.0 & 0.23 & 118 \\
PBE0 & AFM & 5.048 & 0.000 & 0.0 & 0.00 & 123 \\
& FM & 4.990 & 4.227 & 0.0 & 0.09 & 118 \\
HSE [12] & AFM & 5.053 & 0.000 & 0.0 & 0.00 & 151 \\
& FM & 5.013 & 4.800 & 0.0 & 0.04 & - \\
exp. [6] & AFM & 4.978 & - & 0.0 & 0.00 & - \\
& & 4.905 & - & - & - & -
\end{tabular}

are much better in agreement with experiment. Therefore LDA may be more suitable to PuN than GGA. As expected, the lattice constant increases when $U$ or hybrid functional is included. The reason is that the localization of the $5 f$ electrons of $\mathrm{Pu}$ will be enhanced and the cohesion of the crystal is further decreased when the Hubbard $U$ and hybrid functional are introduced. Of all the results, LSDA $+\mathrm{U}$ gives the best lattice constants in AFM phase, deviating by only $0.01 \AA$ from the experimental value. Among the hybrid functionals, Fock- 0.25 yields the very accurate value of $4.876 \AA$ in AFM phase, which is too short by about $0.03 \AA$. Wen et al. [12] obtained the value of $4.978 \AA$ by using the HSE hybrid functional in AFM phase, which is too long by about $0.07 \AA$. According to the calculated band gaps, DFT, DFT $+\mathrm{U}$ and hybrid DFT all predict PuN to be metallic. We have also obtained the values of bulk modulus from the Murnaghan equation of state [24]. Our calculated bulk moduli can be compared to the values $148 \mathrm{GPa}$ and $155 \mathrm{GPa}$ in FM and AFM phases calculated by Fynn and Ray [10] with GGA.

\subsection{Electronic properties and chemical bonding}

Figure 1 shows the calculated density of states (DOS) of $\mathrm{PuN}$ of some selected functionals (LSDA, LSDA $+\mathrm{U}$ and Fock-0.25) in AFM phase. Three cases (LSDA, $\mathrm{LSDA}+\mathrm{U}$ and Fock-0.25) give the same basic features of the electronic spectrum of PuN. There are no band gaps for $\mathrm{PuN}$. The contributions to the total DOS are mainly from $\mathrm{Pu} 5 f$ and $\mathrm{N} 2 p$ states. The $d$ orbitals of $\mathrm{Pu}$ atom contribute less. The DOS in the vicinity of the Fermi level is dominated by the $5 f$ partial DOS. However, the $5 f$ states in the vicinity of the Fermi level are reduced when $U$ and hybrid functional are added. The three main peaks of $5 f$ partial DOS are further separated in the LSDA $+\mathrm{U} /$ Fock- 0.25 scheme than those of
LSDA. These indicates that the localization of $5 f$ electron is highly strengthened in the LSDA $+\mathrm{U} /$ Fock- 0.25 scheme. Now let us compare our calculated DOSs with the valence-band photoelectron spectra. The observed valence-band spectra [9] revealed that the $5 f$ states appear in the vicinity of the Fermi level and three sharp peaks appear within $1 \mathrm{eV}$, located at $0.85 \mathrm{eV}, 0.50 \mathrm{eV}$ and $E_{\mathrm{F}}$. From Fig. 1, no distinct peak is found to the $\mathrm{Pu} 5 f$ states in the range $0-1 \mathrm{eV}$ binding energy in the LSDA $+\mathrm{U}$ scheme. Comparing with LSDA, Fock-0.25 gives a contribution of $\mathrm{Pu} 5 f$ states in the vicinity of the Fermi level which is much smaller. But three peaks appear within $1 \mathrm{eV}$ in both LSDA and Fock-0.25, as is shown in Table II. Therefore on the basis of the lattice constant and density of states, the Fock- 0.25 scheme seems to be more suitable for $\mathrm{PuN}$.

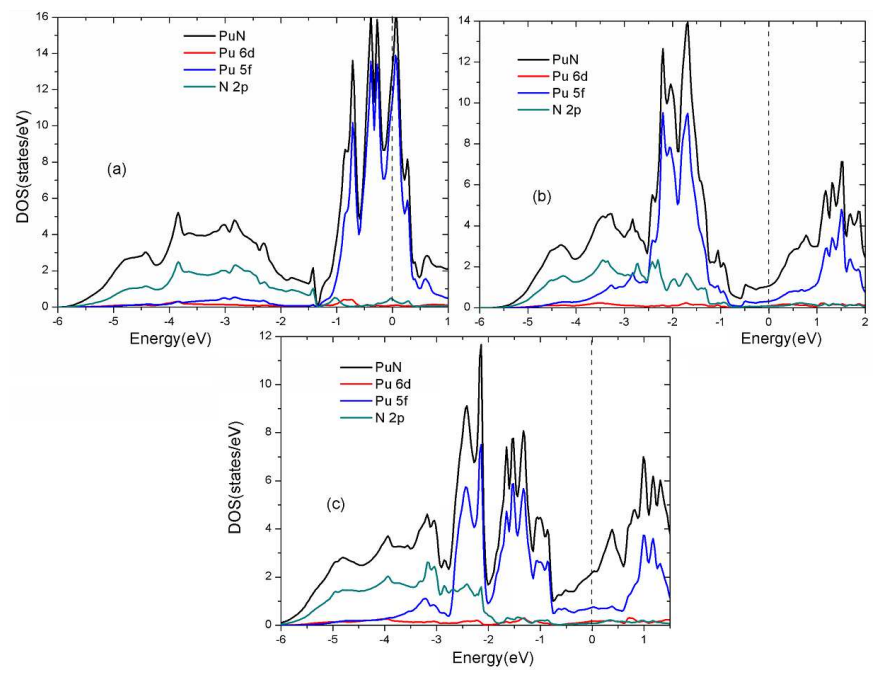

Fig. 1. Total and partial densities of states for AFM PuN in (a) LSDA, (b) LSDA $+\mathrm{U}$, (c) Fock-0.25 calculations.

Three peaks of DOS within $1 \mathrm{eV}$ located TABLE II at $P_{1}, P_{2}$, and $P_{3}[\mathrm{eV}]$ for AFM PuN.

\begin{tabular}{c|c|c|c}
\hline \hline Method & $P_{1}$ & $P_{2}$ & $P_{3}$ \\
\hline LSDA & 0.05 & 0.27 & 0.59 \\
Fock-0.25 & 0.03 & 0.39 & 0.99 \\
Exp. [9] & 0.00 & 0.50 & 0.85
\end{tabular}

In order to obtain further understanding of the electronic structure and analyze the $\mathrm{Pu}-\mathrm{N}$ bonding of $\mathrm{PuN}$, the charge density and the difference charge densities along the (001) plane are plotted in Fig. 2. For the total valence electron density (Fig. 2a), we note high electron density in the immediate vicinity of the nuclei. It reflects that most of the electrons are bound around the atomic nuclei and only a few valence electrons can escape the bondage of the nuclei. For the difference valence electron density (Fig. 2b), the blue and orange zones represent the loss and the gain of charge. We can observe 
that the $\mathrm{N}$ atoms have gained charge and the $\mathrm{Pu}$ atoms lose charge. This clearly suggests that there is net charge transfer from the $\mathrm{Pu}$ atom to the $\mathrm{N}$ atom, indicating that the $\mathrm{Pu}-\mathrm{N}$ bonding has ionic character. This is expected since the $\mathrm{N}$ atom is more electronegative comparing with the $\mathrm{Pu}$ atom. We also note that the charge, to some extent, piles up in the bonding regions between $\mathrm{Pu}$ and $\mathrm{N}$ atoms. This indicates that the $\mathrm{Pu}-\mathrm{N}$ bonding has certain covalent character. The covalent character of the $\mathrm{Pu}-\mathrm{N}$ bonding can be attributed to the hybridization between $\mathrm{Pu} d$ and $\mathrm{N} p$ orbitals (Fig. 3). From Fig. 2b, It is clear that much light charge piles up in the interstices regions away from the bonds. The result suggests that $\mathrm{PuN}$ is metallic as indicated by the DOS analysis.
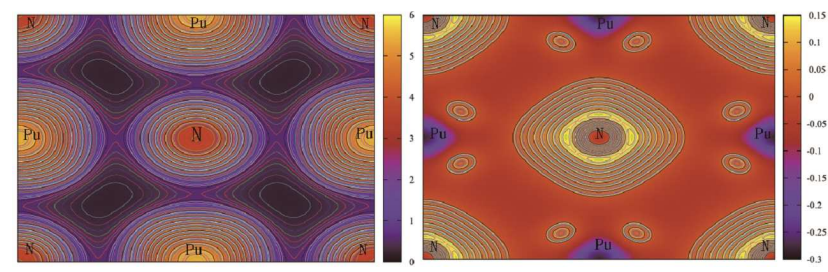

Fig. 2. Contour plots of (left) charge density and (right) charge density difference along the (001) plane in Fock- 0.25 calculations for AFM PuN.

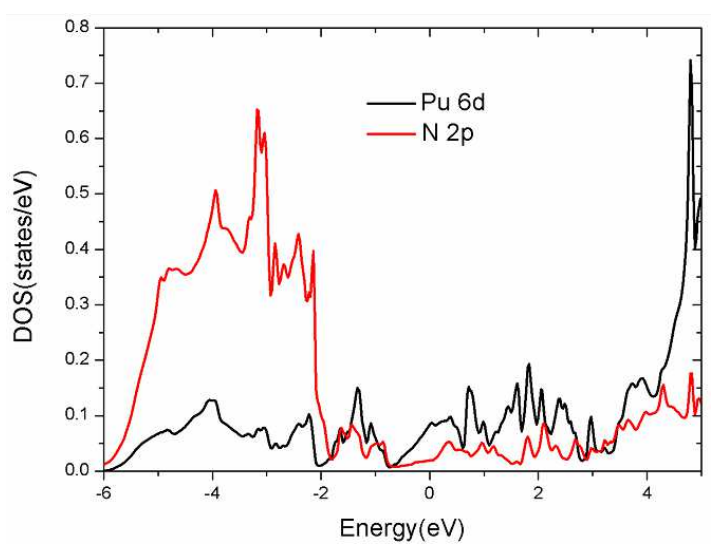

Fig. 3. Partial densities of states for the $\mathrm{Pu} d$ and $\mathrm{N} p$ of AFM PuN in Fock- 0.25 calculations.

Furthermore, we employ a Bader charge analysis [25] to quantify the charge transferred between the $\mathrm{Pu}$ atom and the $\mathrm{N}$ atom. From the value of the charge transferred between $\mathrm{Pu}$ and $\mathrm{N}$ atoms, we can judge the competition between the ionic character and covalent character in PuN. The results obtained in the frameworks of $\mathrm{LSDA}, \mathrm{LSDA}+\mathrm{U}$ and Fock- 0.25 are given in Table III. Since each $\mathrm{Pu}$ atom has 16 electrons outside the core and each $\mathrm{N}$ atom has 5 electrons outside the core, we can find that the loss of the charge of each $\mathrm{Pu}$ atom is transferred to each $\mathrm{N}$ atom in three cases (LSDA, LSDA+U and Fock-0.25). The values of the loss of the charge of each $\mathrm{Pu}$ atom are 1.55|e|, 1.58|e|, and 1.61|e| within
LSDA, LSDA $+\mathrm{U}$ and Fock-0.25 frameworks. This suggests that the ionic character is stronger than covalent character for $\mathrm{Pu}-\mathrm{N}$ bonding. The ionic charges for AFM $\mathrm{PuN}$ can be represented as $\mathrm{Pu}^{+1.55} \mathrm{~N}^{-1.55}$ by LSDA calculations, $\mathrm{Pu}^{+1.58} \mathrm{~N}^{-1.58}$ by $\mathrm{LSDA}+\mathrm{U}$ calculations and $\mathrm{Pu}^{+1.61} \mathrm{~N}^{-1.61}$ by Fock- 0.25 calculations.

Calculated Bader charges $Q_{\mathrm{B}}$ and Bader

TABLE III volumes $V_{\mathrm{B}}$ for AFM PuN.

\begin{tabular}{c|c|c|c|c}
\hline \hline Method & $Q_{\mathrm{B}}(\mathrm{Pu})[e]$ & $Q_{\mathrm{B}}(\mathrm{N})[e]$ & $V_{\mathrm{B}}(\mathrm{Pu})\left[\AA^{3}\right]$ & $V_{\mathrm{B}}(\mathrm{N})\left[\AA^{3}\right]$ \\
\hline LSDA & 14.45 & 6.55 & 17.96 & 11.54 \\
LSDA+U & 14.42 & 6.58 & 17.91 & 11.60 \\
Fock-0.25 & 14.39 & 6.61 & 15.48 & 9.89
\end{tabular}

\subsection{Phonon and thermodynamic properties}

The phonon spectrum is critical for understanding phase stability and thermal transport. Our calculations of phonon spectra of $\mathrm{PuN}$ are based on the supercell approach with the finite displacement method [26]. In this work, the calculations are executed by using LSDA, LSDA $+\mathrm{U}$ and Fock-0.25 schemes. Three cases (LSDA, $\mathrm{LSDA}+\mathrm{U}$ and Fock-0.25) give similar phonon dispersion curves and similar phonon DOS. So for simplicity, Fig. 4 only shows the phonon dispersion curves and phonon DOS obtained by Fock-0.25. Here, the highsymmetry points correspond to $\Gamma(0,0,0), X(0.5,0,0.5)$, $W(0.5,0.25,0.75)$ and $L(0.5,0.5,0.5)$.

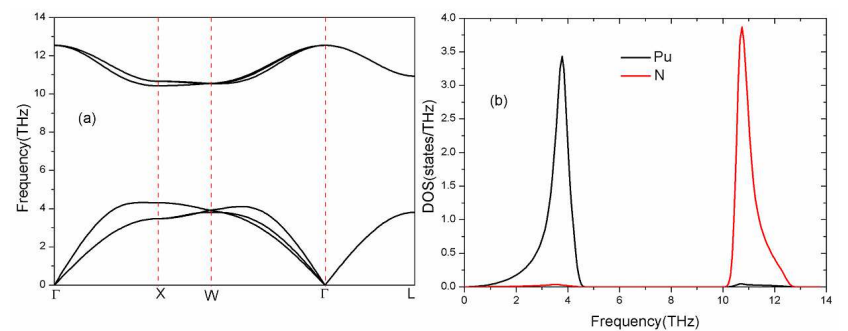

Fig. 4. Calculated (a) phonon dispersion curves and (b) phonon DOS from Fock-0.25 scheme for PuN.

The primitive cell of $\mathrm{PuN}$ has 2 atoms. Therefore, this system contains three acoustic modes and three optical modes. On the basis of the fact that $\mathrm{PuN}$ belongs to $O_{h}$ point group, the irreducible representations of the acoustic modes and optical modes in the center of the BZ are: $\Gamma_{\text {acoustic }}=T_{1 u}$, and $\Gamma_{\text {optical }}=T_{1 u}$, respectively. Here $T_{1 u}$ is infrared-active. As shown in Fig. 4a, the phonon dispersion curves obtained by Fock- 0.25 have no soft mode in the whole Brillouin zone. We can draw the conclusion that $\mathrm{PuN}$ is dynamically stable at ambient pressure and $0 \mathrm{~K}$. The same conclusion can be reached by LSDA and LSDA $+\mathrm{U}$. The phonon frequencies for infrared-active modes $T_{1 u}$ obtained with LSDA, $\mathrm{LSDA}+\mathrm{U}$ and Fock- 0.25 at $\Gamma$ point are $9.63,9.12$, and $12.54 \mathrm{THz}$, respectively. This data need to be checked by future experiments. In the phonon DOS three cases 
(LSDA, LSDA $+\mathrm{U}$ and Fock-0.25) can reach the same conclusion that at lower part, the main contribution to the total phonon DOS is the $\mathrm{Pu}$ atom, and at higher part, it is the $\mathrm{N}$ atom. This difference comes from the fact that the $\mathrm{Pu}$ atom is heavier than the $\mathrm{N}$ atom. For example, the lower part obtained by Fock- 0.25 is from $0 \mathrm{THz}$ to $4.3 \mathrm{THz}$, the higher part obtained by Fock- 0.25 is from $10.4 \mathrm{THz}$ to $12.5 \mathrm{THz}$.

Finally, we investigate the thermodynamic properties of PuN. As is known, thermodynamic properties of solid materials can be evaluated by phonon DOS calculations using the quasi-harmonic approximation (QHA) [27]. The phonon contribution to the Helmholtz free energy $\Delta F$, the entropy $S$, the constant-volume specific heat $C_{v}$ and the phonon contribution to the internal energy $\Delta E$ can be given through the following formulae [28]:

$$
\begin{aligned}
& \Delta F=3 n N k_{\mathrm{B}} T \int_{0}^{\omega_{L}}\left(2 \sinh \left(\frac{\hbar \omega}{2 k_{\mathrm{B}} T}\right)\right) g(\omega) \mathrm{d}(\omega),(3) \\
& S=3 n N k_{\mathrm{B}} \int_{0}^{\omega_{L}}\left[\frac{\hbar \omega}{2 k_{\mathrm{B}} T} \operatorname{coth} \frac{\hbar \omega}{2 k_{\mathrm{B}} T}\right. \\
& \left.-\ln \left(2 \sinh \left(\frac{\hbar \omega}{2 k_{\mathrm{B}} T}\right)\right)\right] g(\omega) \mathrm{d}(\omega), \\
& C_{v}=3 n N k_{\mathrm{B}} \int_{0}^{\omega_{L}}\left(\frac{\hbar \omega}{2 k_{\mathrm{B}} T}\right)^{2} \operatorname{csch}^{2}\left(\frac{\hbar \omega}{2 k_{\mathrm{B}} T}\right) g(\omega) \mathrm{d}(\omega), \\
& \Delta \mathrm{E}=3 n N \frac{\hbar}{2} \int_{0}^{\omega_{L}} \omega \operatorname{coth}\left(\frac{\hbar \omega}{2 k_{\mathrm{B}} T}\right) g(\omega) \mathrm{d}(\omega),
\end{aligned}
$$

where $\omega$ is the phonon frequencies, $g(\omega)$ is the normalized phonon DOS, $N$ is the number of unit cell, $k_{\mathrm{B}}$ is the Boltzmann constant $\hbar$ and is the reduced Planck constant. Figure 5 shows the calculated $\Delta F, S, C_{v}$ and $\Delta E$ of $\mathrm{PuN}$ versus temperatures from $0 \mathrm{~K}$ to $1800 \mathrm{~K}$ in the Fock-0.25 framework. As expected (see Fig. 5b), the vibrational entropy curves increase smoothly as temperature increases because the vibrational frequencies increase with temperature. From Fig. $5 \mathrm{c}$, the $C_{v}$ increases very rapidly with $T^{3}$ at sufficiently low temperatures, and almost approaches the Dulong-Petit limit when the temperature is higher than about $1000 \mathrm{~K}$. The DulongPetit law [29] is $C_{v}=3 N R$, where $N$ and $R$ refer to the number of atoms and the gas constant. For PuN, $N$ equals 2 , which means the theoretical value of $C_{v}$ should be $49.9 \mathrm{~J} /(\mathrm{mol} \mathrm{K})$. While our calculated $C_{v}$ tends to be a constant of $49.7 \mathrm{~J} /(\mathrm{mol} \mathrm{K})$ at high temperature. Obviously, the deviation is only $0.4 \%$.

Table IV lists our calculated temperature variations of the entropy values $S$ and the enthalpy difference $H-H_{298}$, comparison with previous thermodynamic data given by Oetting [7]. We obtain the enthalpy values from the equation: $\Delta H=\Delta E+P \Delta V$, where $E, P$, and $V$ refer to the internal energy, the pressure and the volume, respectively [30]. In solid and liquid phase, the change in term $P \Delta V$ is very small compared to $\Delta E$. The equilibrium pressure is set to be zero in our calculations. Therefore, $\Delta H \approx \Delta E$. Our calculated $S$ and $H-H_{298}$ from LSDA,

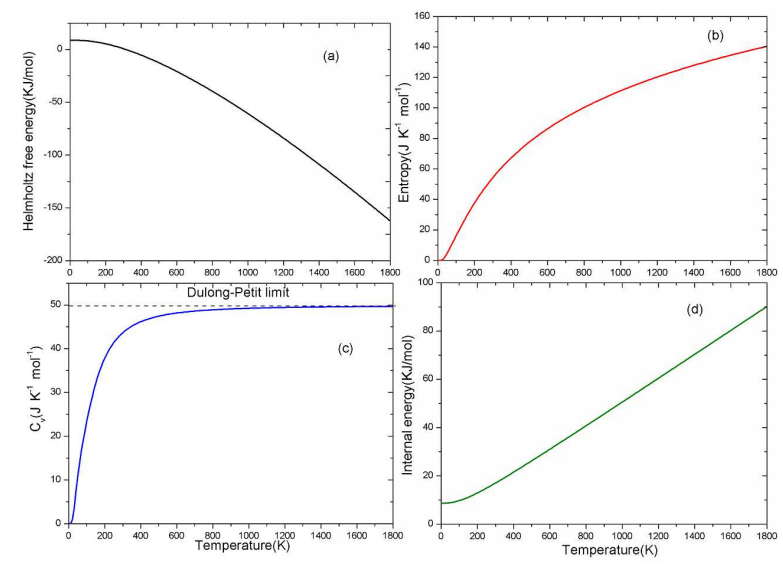

Fig. 5. Calculated (a) the Helmholtz free energy $\Delta F$, (b) the entropy $S$, (c) the constant-volume specific heat $C_{v},(\mathrm{~d})$ the internal energy $\Delta E$ of $\mathrm{PuN}$ in the Fock-0.25 framework.

$\mathrm{LSDA}+\mathrm{U}$ and Fock- 0.25 are close to the previous data. The lower the temperature is, the more close to the previous data the results are. The slightly larger deviation under high temperature may be due to the limitation of the QHA, because the anharmonic effect between the phonon is not taken into account. The high-order anharmonicity at high temperature may become more prominent than at low temperature for PuN. Besides the limitation of the QHA, the electronic contribution to the entropy is ignored in our calculations. We note that the Fock-0.25 values is closest to the values given by Oetting [7], compared to the LSDA and LSDA $+\mathrm{U}$ values.

TABLE IV

Our calculated $S-S_{298}[\mathrm{~J} /(\mathrm{mol} \mathrm{K})]$ and $H-H_{298}[\mathrm{KJ} / \mathrm{mol}]$ of $\mathrm{PuN}$, along with other theoretical values.

\begin{tabular}{c|c|c|c|c}
\hline \hline$T[\mathrm{~K}]$ & LSDA & LSDA $+\mathrm{U}$ & Fock-0.25 & Other [7] \\
\hline \multicolumn{5}{|c}{$S[\mathrm{~J} /(\mathrm{mol})]$} \\
\hline 300 & 54.20 & 59.57 & 60.89 & 59.69 \\
400 & 67.15 & 72.93 & 74.29 & 75.55 \\
500 & 77.61 & 83.59 & 84.96 & 88.50 \\
600 & 86.33 & 92.43 & 93.81 & 99.43 \\
700 & 93.79 & 99.96 & 101.35 & 108.88 \\
800 & 100.30 & 106.52 & 107.91 & 117.19 \\
900 & 106.07 & 112.32 & 113.72 & 124.62 \\
1000 & 111.25 & 117.53 & 118.92 & 131.34 \\
$T[\mathrm{~K}]$ & LSDA & LSDA+U & Fock-0.25 & Other [7] \\
\hline \multicolumn{5}{|c}{$H-H_{298}[\mathrm{KJ} / \mathrm{mol}]$} \\
\hline 300 & 0.08 & 0.09 & 0.09 & 0.10 \\
400 & 4.59 & 4.74 & 4.75 & 5.62 \\
500 & 9.28 & 9.52 & 9.54 & 11.43 \\
600 & 14.06 & 14.37 & 14.39 & 17.43 \\
700 & 18.90 & 19.25 & 19.28 & 23.55 \\
800 & 23.78 & 24.16 & 24.19 & 29.78 \\
900 & 28.68 & 29.09 & 29.12 & 36.09 \\
1000 & 33.60 & 34.03 & 34.07 & 42.47
\end{tabular}




\subsection{Spin-orbit coupling effects}

In this section, we will investigate spin-orbit coupling (SOC) effects on the structural, magnetic, and electronic properties of PuN. The values of energy difference $\Delta E$ (eV per PuN) between the FM and AFM states obtained at the SOC level are listed in Table V. We find that the FM phase is stable within the LSDA+SOC approach. It is similar to the case in our previous LSDA studies, indicating that the inclusion of SOC cannot alter the magnetic ordering of $\mathrm{PuN}$. The results from the LSDA $+\mathrm{U}+\mathrm{SOC}$ and Fock- $0.25+\mathrm{SOC}$ schemes illustrate that the $\mathrm{PuN}$ is AFM, which is consistent with our previous NSOC calculations and experimental result [8]. Comparing the results obtained at the SOC

\section{TABLE V}

Structural properties of PuN: lattice constant a [§], $\Delta E=E_{\mathrm{FM}}-E_{\mathrm{AFM}}[\mathrm{eV} /$ per $\mathrm{PuN}]$ and bulk modulus $B$ [GPa] with SOC calculations. The Hubbard parameter $U$ is $0.29 \mathrm{Ry}$.

\begin{tabular}{c|c|c|c|c}
\hline \hline Method & magn. & $a[\AA]$ & $\Delta E[\mathrm{eV}]$ & $B[\mathrm{GPa}]$ \\
\hline \multirow{2}{*}{ LSDA+SOC } & FM & 4.852 & -0.07 & 181 \\
& AFM & 4.840 & 0.00 & 189 \\
LSDA+U+SOC & FM & 5.064 & 0.11 & 167 \\
& AFM & 4.953 & 0.00 & 152 \\
Fock-0.25+SOC & FM & 4.855 & 0.13 & 199 \\
& AFM & 4.877 & 0.00 & 210
\end{tabular}

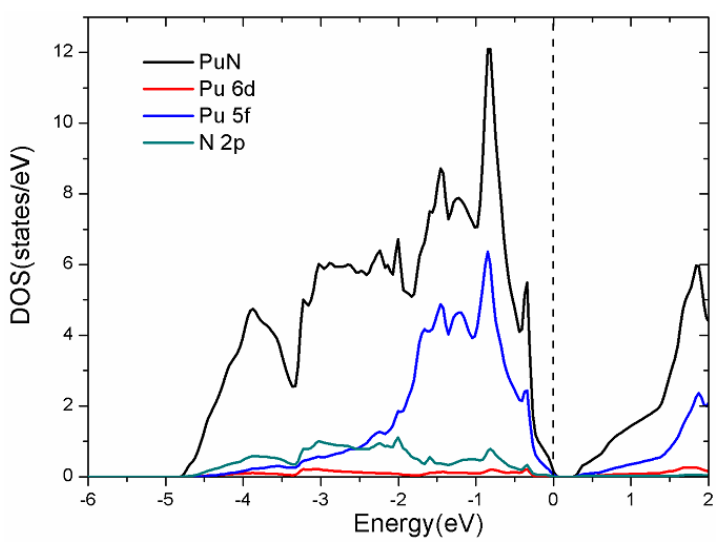

Fig. 6. Total and partial densities of states for AFM $\mathrm{PuN}$ in $\mathrm{LSDA}+\mathrm{U}+\mathrm{SOC}$ calculations.

(Table V) and NSOC (Table I) levels, we observe the same trend that the lattice constant is slightly increased from NSOC to SOC calculations. For example, the result from $\mathrm{LSDA}+\mathrm{U}+\mathrm{SOC}$ of $\mathrm{PuN}$ in $\mathrm{AFM}$ phase is $a_{0}=4.953 \AA$, which reports an increase of the lattice constant of $0.037 \AA$ with respect to the LSDA $+\mathrm{U}$ scheme. Therefore for PuN, the discrepancy between the experiment and our calculation increases from $0.22 \%$ for $\mathrm{LSDA}+\mathrm{U}$ to $0.98 \%$ for $\mathrm{LSDA}+\mathrm{U}+\mathrm{SOC}$. To check the effect of SOC on the bulk modulus, we calculate the bulk modulus at the SOC level. The bulk modulus tends to decrease when the SOC calculations are added in the LSDA $+\mathrm{U}$ and Fock- 0.25 schemes, while it increases within LSDA+SOC compared with that in the LSDA. For example, LSDA $+\mathrm{U}+\mathrm{SOC}$ gives a value of $152 \mathrm{GPa}$ in AFM phase which is slightly lower than the value $(172 \mathrm{GPa})$ obtained with LSDA + U. Furthermore, prominent changes happen in the DOS calculations when the SOC effect is considered. From Fig. 6, we can observe that there is a zero occupation of $\mathrm{Pu} 5 f$ states at the Fermi level in the LSDA $+\mathrm{U}+\mathrm{SOC}$ scheme, which has disagreement with a photoelectron spectroscopy study of valence-band spectra [9]. Hence the LSDA $+\mathrm{U}+\mathrm{SOC}$ scheme predicts a semiconductive state for PuN. However, LSDA $+\mathrm{U}$ calculations predict a metallic ground state for $\mathrm{PuN}$.

\section{Conclusions}

we have performed DFT, DFT $+\mathrm{U}$ and hybrid DFT calculations on the structural, magnetic, electronic, dynamical, and thermodynamic properties of $\mathrm{PuN}$. The correct ground-states of AFM phase PuN are successfully reached by DFT $+\mathrm{U}$ and hybrid DFT. Our calculated lattice constants are in good accordance with the experimental data. In particular, Fock- 0.25 yields very accurate lattice constant in AFM phase (typically with a deviation of $0.03 \AA$ ). Calculated electronic density of states show the important contributions of $\mathrm{Pu} 5 f$ states to the Fermi-level occupation. Three peaks in the range $0-1 \mathrm{eV}$ binding energy can be found in LSDA and Fock0.25 . According to our charge density differences and Bader charge analysis, the bonds for PuN have a mixture of covalent and ionic components, but the ionic character is stronger than covalent character. The calculations of phonon spectra of PuN have been performed, showing that $\mathrm{PuN}$ is dynamically stable at ambient pressure and $0 \mathrm{~K}$. The infrared active frequencies are presented. Furthermore, the thermodynamic properties such as $\Delta F, S$, $C_{v}$ and $\Delta E$ are studied from $0 \mathrm{~K}$ to $1800 \mathrm{~K}$ within the QHA. The calculated values of $S$ and $H-H_{298}$ are close to previous thermodynamic data given by Oetting. Finally, the effect of spin-orbit coupling on the structural, magnetic, and electronic properties of $\mathrm{PuN}$ has been discussed. We expect that these calculated results will be useful for the application of PuN in the generation-IV reactor and nuclear industry.

\section{Acknowledgments}

The authors would like to thank ShiZhang Li and XiangGang Kong for helpful comments and discussions.

\section{References}

[1] The Chemistry of the Actinide and Transactinide Elements, Vols. 1-5, Eds. L.R. Morss, N.M. Edelstein, J. Fuger, J.J. Katz, Springer, New York 2006. 
[2] Actinides-Basic Science, Applications, and Technology, Eds. L. Soderholm, J.J. Joyce, M.F. Nicol, D.K. Shuh, J.G. Tobin, MRS Symposia Proceedings No. 802, Materials Research Society, Pittsburgh 2004.

[3] Actinides 2005: Basic Science, Applications, and Technology, Eds. J.L. Sarrao, A.J. Schwartz, M.R. Antonio, P.C. Burns, R.G. Haire, H. Nitsche, MRS Symposia Proceedings No. 893, Materials Research Society, Pittsburgh 2005.

[4] F. Brown, H.M. Ockenden, G.A. Welch, J. Chem. Soc. 4196 (1955).

[5] W.M. Pardue, V.W. Storhok, R.A. Smith, P.H. Bonnell, J.F. Gates, D.L. Keller, Synthesis, Fabrication and Chemical Reactivity of Plutonium Mononitride, Battelle Memorial Institute, Columbus, Ohio, BMI1693, 1964.

[6] V.J. Tennery, E.S. Bomar, J. Am. Ceram. Soc. 54, 247 (1971).

[7] F.L. Oetting, The Chemical Thermodynamic Properties of Plutonium Compounds, The Dow Company, Rochy Flats Division, Golden, Colorado 1996.

[8] R.O.A. Hall, J.A. Lee, D.J. Martin, M.J. Mortimer P.W. Sutcliffe, J. Chem . Thermodyn. 10, 935 (1978).

[9] L. Havela, F. Wastin, J. Rebizant, T. Gouder, Phys. Rev. B. 68, 085101 (2003).

[10] R.A. Fynn, A.K. Ray, Phys. Rev. B 76, 115101 (2007).

[11] L. Petit, A. Svane, W.M. Temmerman, Z. Szotek, Phys. Rev. B 80, 045124 (2009).

[12] X.D. Wen, R.L. Martin, G.E. Scueria, S.P. Rudin, E.R. Batista, J. Phys. Chem. C 117, 13122 (2013).

[13] H. Wang, K. Konashi, J. Alloys Comp. 533, 53 (2012).

[14] H. Nakamura, M. Machida, M. Kato, Phys. Rev. B 82, 155131 (2010).
[15] K. Schwarz, P. Blaha, G.K.H. Madsen, Comput. Phys. Commun. 147, 71 (2002); P. Blaha, K. Schwarz, G.K.H. Madsen, D. Kvasnicka, J. Luitz, WIEN2K, An Augmented Plane Wave Local Orbitals Program for Calculating Crystal Properties, Technische Universität Wien, Austria 2001.

[16] P. Blaha, K. Schwarz, P.I. Sorantin, S.B. Trickey, Comput. Phys. Commun. 59, 399 (1990).

[17] M. Petersen, F. Wagner, L. Hufnagel, M. Scheffler, P. Blaha, K. Schwarz, Comput. Phys. Commun. 126, 294 (2000).

[18] P. Novák, J. Kuneš, L. Chaput, W.E. Pickett, Phys. Status Solidi B 243, 463 (2006).

[19] F. Tran, P. Blaha, K. Schwarz, P. Novák, Phys. Rev. B 74, 155108 (2006).

[20] M. Ernzerhof, G.E. Scuseria, J. Chem. Phys. 110 , 5029 (1999)

[21] C. Adamo, V. Barone, J. Chem. Phys. 110, 6158 (1999).

[22] I. de P.R. Moreira, F. Illas, R.L. Martin, Phys. Rev. B 65, 155102 (2002).

[23] J. Kuneš, P. Novak, M. Diviš, P.M. Oppeneer, Phys. Rev. B 63, 205111 (2001).

[24] F.D. Murnaghan, Proc. Natl. Acad. Sci. USA 30, 244 (1944).

[25] R.F.W. Bader, Atoms in Molecules: A Quantum Theory, Oxford University Press, New York 1990.

[26] A. Togo, F. Oba, I. Tanaka, Phys. Rev. B 78, 134106 (2008).

[27] A. Siegel, K. Parlinski, U.D. Wdowik, Phys. Rev. B 74, 104116 (2006).

[28] C. Lee, X. Gonze, Phys. Rev. B 51, 8610 (1995).

[29] A.T. Petit, P.L. Dulong, Ann. Chim. Phys. 10, 395 (1819), (in French).

[30] L. Sukit, J. Sirichok, Phys. Rev. B 70, 054104 (2004). 